\title{
SPATIAL WAVELET PACKET DENOISING FOR IMPROVED DOA ESTIMATION
}

\author{
R. Sathish and G. V. Anand \\ Department of Electrical Communication Engineering \\ Indian Institute of Science \\ Bangalore-560012, INDIA \\ Phone: +918022932277 \\ E-mail: sathish@protocol.ece.iisc.ernet.in, anandgv@ece.iisc.ernet.in
}

\begin{abstract}
The performance of direction-of-arrival (DOA) estimation techniques such as MUSIC degrades progressively with decreasing signal-to-noise ratio (SNR). The DOA estimation performance may be improved by employing a pre-processor that enhances the SNR, before performing the DOA estimation. In this paper, a denoising technique based on the use of wavelet packet transform in the spatial domain is proposed for enhancing the output SNR of a uniform linear array of sensors receiving narrowband signals in the form of plane waves from different directions. The technique involves the use of a spatial wavelet packet transform (SWPT) followed by a block thresholding scheme based on the norm of SWPT subvectors in different spatial frequency subbands. This method has the advantage of not requiring the high sampling rates demanded by the temporal wavelet denoising techniques. It is shown through simulations that SWPT denoising (SWD) requires a sampling rate that is just 2-4 times the signal frequency, whereas temporal wavelet denoising (TWD) requires a much higher sampling rate for achieving a comparable SNR gain. Consequently, at lower sampling rates, the DOA estimation performance indices, such as bias, mean square error and resolution, achieved by SWD are much superior to those achieved by TWD or by undenoised data.
\end{abstract}

\section{INTRODUCTION}

Wavelet denoising is a nonlinear signal enhancement technique that involves the application of a wavelet transform to a noisy signal followed by thresholding of the wavelet coefficients to eliminate much of the noise without causing a significant distortion of the signal $[1,2]$. A cleaner version of the signal is recovered by applying the inverse wavelet transform of the vector of 
thresholded wavelet coefficients. This denoising scheme may be applied to the output of each sensor of a multi-sensor array to enhance the SNR at the array. Still greater enhancement of the array SNR can be achieved by using the multi-sensor denoising technique of Rao and Jones [3] that exploits the inter-sensor correlation or spatial correlation of signals at different sensors in the array.

In one version of the multi-sensor denoising technique, called the DFTWT filter, the multi-sensor signals are subjected to approximate spatial decorrelation through the application of spatial DFT to each snapshot of the array data vector before performing wavelet denoising in the time domain and spatial recorrelation through the application of inverse spatial DFT $[3,4]$. One interesting application of the multi-sensor denoising is for denoising the data received by an array of sensors used for direction-of-arrival (DOA) estimation. In the context of DOA estimation by MUSIC [6], it has been shown that the application of multi-sensor wavelet denoising to the sensor array data prior to DOA estimation leads to a significant improvement in the performance of the MUSIC estimator $[4,5]$. All the denoising techniques discussed above, including the DFT-WT filter, may be called temporal wavelet denoising (TWD) techniques since the wavelet transform is applied to the signals in the time domain. In TWD techniques, a high sampling rate of at least 12 times the signal frequency has to be employed to achieve a significant SNR gain.

In this paper, a spatial wavelet denoising (SWD) technique of denoising array data is presented. In the SWD technique the array data is subjected to a spatial wavelet packet transform (SWPT), which divides the entire spatial frequency range into uniform subbands. The filtering is done by an adaptive block thresholding method, wherein the norm of the SWPT coefficient vector in each subband is compared with an estimate of the noise variance in the subband. This process of thresholding achieves denoising in the spatial domain by eliminating noise from those angular sectors (spatial frequency subbands) that do not contain signal. Performing an inverse SWPT yields the SNR-enhanced multi-sensor signal. The primary advantage of this SWD technique is that it achieves SNR gain comparable to that of the DFT-WT filter at a much lower sampling rate of just $2-4$ times the signal frequency. Also, SWD eliminates the computation of spatial DFT's and IDFT's required by the DFT-WT filter. Thus, SWD achieves a reduction in both the hardware complexity and computational complexity of the array denoising. Simulation results are presented to illustrate the improvement in the performance of the MUSIC DOA estimator brought about by the application of SWD to the array data before performing DOA estimation.

\section{DATA MODEL}

Consider a uniform linear array (ULA) of $M$ sensors with intersensor spacing $d$. If plane waves from $J$ narrowband sources with center frequency $\frac{\omega}{2 \pi}$ arrive 
at the array at angles $\theta_{1}, \theta_{2}, \ldots, \theta_{J}$, with respect to the array normal, the output of the $m^{\text {th }}$ sensor can be written as

$$
\begin{aligned}
& x_{m}(t)=s_{m}(t)+n_{m}(t), \\
& s_{m}(t)=\sum_{p=1}^{J} b_{p}(t) e^{j \omega t+j(m-1) k d \sin \left(\theta_{p}\right)},
\end{aligned}
$$

where $b_{p}(t)$ is the slowly varying zero-mean complex random amplitude of the signal from the $p^{\text {th }}$ source, $k$ is the wavenumber and $n_{m}(t)$ is zero-mean additive white complex Gaussian noise of variance $\sigma^{2}$. It is assumed that the signal and noise are mutually uncorrelated and that $J \leq M$. If we write $\mathbf{x}(t)=\left[\begin{array}{llll}x_{1}(t) & x_{2}(t) & \ldots & x_{M}(t)\end{array}\right]^{T}$, then

$$
\mathbf{x}(t)=\mathbf{A b}(t)+\mathbf{n}(t)
$$

where $\mathbf{n}(t)=\left[n_{1}(t) n_{2}(t) \ldots n_{M}(t)\right]^{T}$ is the noise vector that is also spatially white, and

$$
\mathbf{b}(t)=\left[b_{1}(t) e^{j \omega t} b_{2}(t) e^{j \omega t} \ldots b_{J}(t) e^{j \omega t}\right]^{T},
$$

is the vector of source signals at time $t$. The steering-vector matrix $\mathbf{A}$ is given by

$$
\mathbf{A}=\left[\begin{array}{lll}
\mathbf{a}\left(\theta_{1}\right) & \mathbf{a}\left(\theta_{2}\right) & \ldots \\
\mathbf{a}\left(\theta_{J}\right)
\end{array}\right],
$$

where

$$
\mathbf{a}(\theta)=\left[1 e^{j k d \sin (\theta)} e^{j 2 k d \sin (\theta)} \ldots e^{j(M-1) k d \sin (\theta)}\right]^{T} .
$$

It is assumed that the sources are mutually uncorrelated and that the autocorrelation of the signal from each source decays exponentially with the same decay rate, i.e.,

$$
E\left[b_{p}(t) b_{q}^{*}(u)\right]=\sigma_{p}^{2} \delta_{p, q} e^{-\alpha|t-u|} .
$$

\section{SPATIAL POWER SPECTRAL DENSITY (SPSD)}

Let us stack the received data from all sensors into a $M \times 1$ column vector for a snapshot at time $t$ as

$$
\mathbf{x}(t)=\left[x_{1}(t) x_{2}(t) \ldots x_{M}(t)\right]^{T} .
$$

Thus,

$$
\mathbf{x}(t)=\mathbf{s}(t)+\mathbf{n}(t)
$$

where $\mathbf{s}(t)$ and $\mathbf{n}(t)$ are similar $M \times 1$ snapshot vectors corresponding to signal and noise respectively.

The power spectrum of the vector $\mathbf{x}(t)$ would contain $n$ distinct peaks corresponding to $n$ distinct DOAs [7], which ride over a plateau of height equal to the noise variance $\sigma^{2}$ (noise is spatially white). Figure 1 shows three peaks of the SPSD corresponding to the three sources at angles $\theta_{1}=10^{\circ}$, $\theta_{2}=15^{\circ}$ and $\theta_{3}=20^{\circ}$ with respect to the broadside of the ULA. The 


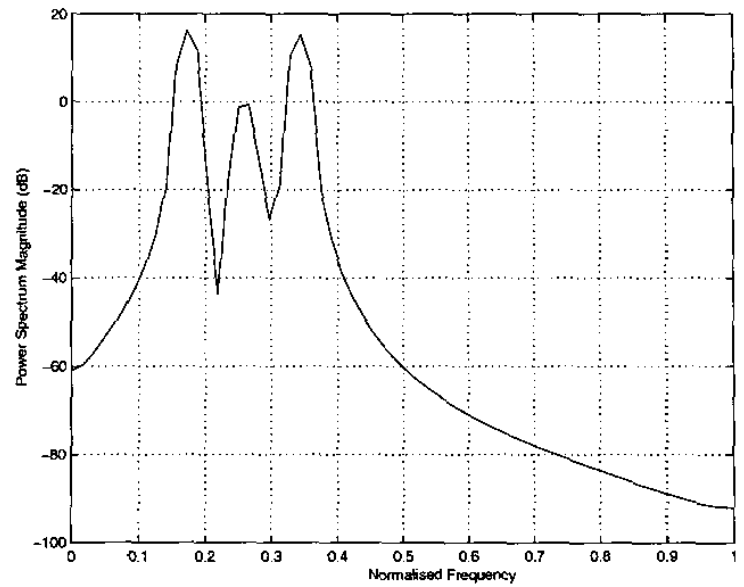

Figure 1: SPSD of one realisation of noiseless data vector.

SPSD was calculated for one realisation of the noisefree signal. The peaks are located at the normalised spatial frequency $\omega_{\text {norm }}$ which is related to the direction $\theta$ as $\omega_{\text {norm }}=\sin (\theta \pi / 180)$, where $\theta$ is measured in degrees.

\section{DISCRETE WAVELET PACKET TRANSFORM (DWPT)}

The Discrete Wavelet Packet Transform is a generalised form of the wavelet transform wherein the process of filtering and down-sampling [8] is applied to the detail coefficients also at each level of decomposition. This process allows decomposition into uniform bands in the frequency domain. The filter bank structure of a three level DWPT is like a full binary tree as seen in Figure 2. The filters $g(-n)$ and $h(-n)$ are lowpass and highpass filters respectively. The frequency response of the wavelet packet filter bank is shown in Figure 3. The changes in the order of the output bands in Figure 3 is explained by the fact that a highpass filter flips the resulting spectrum [9]. The size of each frequency band is the same and therefore the amount of white-noise energy contained in each band would also be the same. If the input to the filter bank consists of a multi-frequency signal buried in white noise, the energy in the subbands containing the signal would be greater than that in the subbands containing noise only. Hence, the noise in the subbands that do not contain any signal component can be removed by thresholding. 


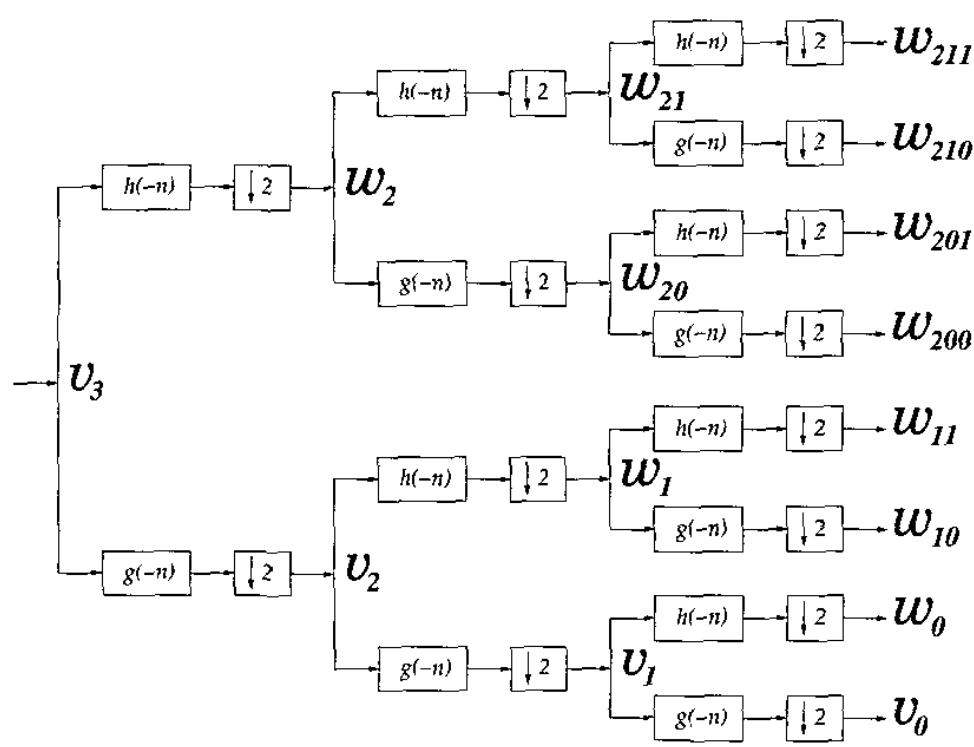

Figure 2: Binary Tree Filter Bank Structure: Full Packet Decomposition

\section{SWD USING SWPT}

A DWPT applied in the spatial domain to the multi-sensor signal becomes a spatial wavelet packet transform (SWPT). The SWPT divides the entire spatial frequency range into frequency bands of uniform width. The method of SWD adopted in this work is based on an empirical energy comparison algorithm whereby the spatial frequency bands that contain the signal are identified and the corresponding SWPT coefficients are retained. The SWPT coefficients in the bands that do not contain signal energy are discarded, which results in a significant reduction of noise energy in the received data.

Let $\mathbf{W}_{\mathbf{p}, l}$ represent a $l$-level SWPT matrix and let $\mathbf{x}_{\mathbf{p}, l}(t)=\mathbf{W}_{\mathbf{p}, l}[\mathbf{x}(t)]$, $\mathbf{s}_{\mathbf{p}, l}(t)=\mathbf{W}_{\mathbf{p}, l}[\mathbf{s}(t)]$ and $\mathbf{n}_{\mathbf{p}, l}(t)=\mathbf{W}_{\mathbf{p}, l}[\mathbf{n}(t)]$ be $M \times 1$ column vectors containing SWPT coefficients of received data, signal and noise respectively. The subscript ' $p$ ' in the symbols for column vectors stand for the SWPT coefficients. Since SWPT is a linear operation,we have

$$
\mathbf{x}_{\mathbf{p}, l}(t)=\mathbf{s}_{\mathbf{p}, l}(t)+\mathbf{n}_{\mathbf{p}, l}(t) .
$$

Let the SWPT coefficients be segregated into coefficients corresponding to different frequency bands as, $\mathbf{x}_{\mathbf{p}, l}(t)=\left[\mathbf{x}_{\mathbf{p}, l}^{1}(t) \mathbf{x}_{\mathrm{p}, l}^{2}(t) \ldots \mathbf{x}_{\mathbf{p}, l}^{m}(t)\right]$, where $\mathbf{x}_{\mathbf{p}, l}^{i}(t)$ corresponds to the set of SWPT coefficients at level $l$ in the $i^{\text {th }}$ spatial frequency band, and $m$ is the total number of frequency bands at level $l$, where $m=2^{l}$. Similarly, $\mathbf{s}_{\mathbf{p}, l}^{i}(t)$ and $\mathbf{n}_{\mathrm{p}, l}^{i}(t)$ correspond to the sets of signal and noise coefficients in the $i^{\text {th }}$ frequency band, respectively. 


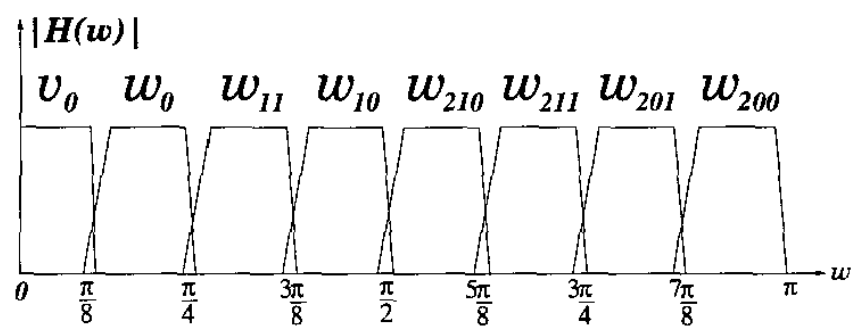

Figure 3: Frequency bands for the Binary Tree decomposition.

The presence of a signal peak in the $i^{\text {th }}$ band can be detected by using the norm of the SWPT data subvector, $\mathbf{x}_{\mathbf{p}, l}^{i}(t)$. If we start with $M$ sensor outputs (spatial data samples) at level 0 , then at level $l$ we have $\frac{M}{2^{l}}$ coefficients in each spatial frequency band. Thus, at level $l$, if a band contains both signal and noise, we can write

$$
\begin{aligned}
E\left[\left\|\mathbf{x}_{\mathbf{p}, l}^{i}(t)\right\|^{2}\right] & =E\left[\left\|\mathbf{s}_{\mathbf{p}, l}^{i}(t)\right\|^{2}\right]+E\left[\left\|\mathbf{n}_{\mathbf{p}, l}^{i}(t)\right\|^{2}\right], \\
& =E\left[\left\|\mathbf{s}_{\mathbf{p}, l}^{i}(t)\right\|^{2}\right]+\frac{M}{2^{l}} \sigma^{2},
\end{aligned}
$$

where $E$ is the expectation operator. Hence,

$$
E\left[\left\|\mathbf{x}_{\mathbf{p}, l}^{i}(t)\right\|^{2}\right] \geq \frac{M}{2^{l}} \sigma^{2} i=1,2, \ldots, 2^{l}
$$

In the relation above, the equality holds if and only if the $i^{\text {th }}$ frequency band does not contain a signal component. Thus, based on a comparison of the norm of the subvector $\mathrm{x}_{\mathrm{p}, l}^{i}(t)$ with a threshold, a decision can be made as to whether the $i^{\text {th }}$ spatial frequency band contains signal energy or not.

In reality, one can only have an estimate of the noise standard deviation $\sigma$. A reliable estimate $\hat{\sigma}$ can be obtained from the median $m_{\mathbf{x}}$ of the first level detail coefficients of a temporal wavelet transform of the sensor output $[1,2]$,

$$
\hat{\sigma}=\frac{m_{\mathbf{x}}}{0.6745} .
$$

Also, the expectation in (12) is estimated by averaging over $N$ snapshots of the array data,

$$
E\left[\left\|\mid \mathrm{x}_{\mathrm{p}, l}^{i}(t)\right\|^{2}\right] \approx \frac{1}{N} \sum_{t=1}^{N}\left\|\mathrm{x}_{\mathrm{p}, l}^{i}(t)\right\|^{2} .
$$

Hence, the following decision rule may be adopted to decide whether the $i^{\text {th }}$ frequency band contains a signal component or not:

If

$$
\frac{1}{N} \sum_{t=1}^{N}\left\|\mathrm{x}_{\mathrm{p}, l}^{i}(t)\right\|^{2}>\beta \frac{M}{2^{l}} \hat{\sigma}^{2}
$$


decide signal component is present, otherwise, decide signal component is not present.

The quantity $\beta$ is an empirical tolerance parameter slightly greater than 1. The inclusion of the parameter $\beta$ is necessitated by the fact that as the number of levels $l$ in the SWPT is increased, the number of coefficients in a band decreases logarithmically, and hence the quantity $\frac{M}{2^{2}} \hat{\sigma}^{2}$ may under estimate the noise energy in the band. Denoising is achieved by discarding all the SWPT coefficients in all frequency bands that do not contain a signal component. Thus, we arrive at the following algorithm.

\section{Algorithm: SWD using SWPT}

Step 1. Perform a single-level SWPT on the array data to obtain $\mathrm{x}_{\mathrm{p} .1}(t), t=$ $1,2, \ldots, N$.

Step 2. Calculate $\left(\frac{1}{N} \sum_{t=1}^{N}\left\|\mathbf{x}_{\mathrm{p}, 1}^{i}(t)\right\|^{2}\right)$ for $i=1,2$ and compare the two norm averages with $\beta \frac{M}{2^{T}} \hat{\sigma}^{2}$.

If for a particular band $i$ the norm average is less than or equal to $\beta \frac{M}{2^{1}} \hat{\sigma}^{2}$, then discard $\mathrm{x}_{\mathrm{p} .1}^{i}(t)$ coefficients in that band $i$.

Else, if the norm average exceeds $\beta \frac{M}{2^{\tau}} \hat{\sigma}^{2}$, go to Step 1 , replace $M$ by $\frac{M}{2}$, and perform another single-level SWPT on $\mathbf{x}_{\mathrm{p}, 1}^{i}(t)$ for that band $i$ to obtain $\mathbf{x}_{\mathrm{p}, 2}^{i}(t)$.

Repeat steps 1,2 until a predefined level $l$ of the SWPT is reached.

Step 3. Perform an inverse SWPT to obtain the snapshots of the denoised array data vector.

\section{SIMULATIONS}

Narrowband signals of equal strength from three sources with a common center frequency $\frac{\omega}{2 \pi}$ were assumed to be incident in the form of plane waves on a ULA of $M=45$ sensors with inter-element spacing $d=\frac{\lambda}{2}$. The directions of arrival were chosen to be $\theta_{1}=60^{\circ}, \theta_{2}=66^{\circ}$ and $\theta_{3}=72^{\circ}$ with respect to the broadside of the array. Spatially and temporally white Gaussian noise was added to the signal so that the received data has the desired SNR. The source decorrelation parameter $\alpha$ in (7) was set to $\frac{1}{70}$ and a total of $N=128$ snapshots of data was collected. The SWD and TWD were performed using $d b 16$ wavelet.

To study the improvement in signal quality, an empirical SNR gain function $G$, defined below

$$
G=S N R_{o u t}-S N R_{i n},
$$




$$
\begin{aligned}
S N R_{\text {out }} & =10 \log _{10}\left(\frac{E\left[\|\mathbf{S}\|^{2}\right]}{E\left[\|\mathbf{S}-\widehat{\mathbf{S}}\|^{2}\right]}\right), \\
S N R_{\text {in }} & =10 \log _{10}\left(\frac{E\left[\|\mathbf{S}\|^{2}\right]}{E\left[\|\mathbf{S}-\mathbf{X}\|^{2}\right]}\right),
\end{aligned}
$$

has been numerically computed and plotted in Figure 4 for varying SNR of received data. The quantities $\mathbf{S}$ and $\mathbf{X}$ are respectively, the array signal vector and the received array data vector (each of length $M N$ ), obtained by stacking the temporal output vectors of all sensors one below the other. Similarly, $\hat{\mathbf{S}}$ is the spatially denoised array signal vector. For computing $S N R_{\text {out }}$ and $S N R_{\text {in }}$ in (17) and (18), the expectations were replaced by averaging over 100 Monte-Carlo simulations. The trend of the plot in Figure 4 shows that the SWD achieves significant signal enhancement at lower SNRs while the gain tends to decrease with increasing SNR. This could be due to the fact that, at higher $\mathrm{SNR}$, the thresholding process may remove some signal energy also, in addition to removing noise.

The performance of the SWD and the TWD schemes at $4 \mathrm{~dB}$ input SNR for different values of the sampling frequency-to-signal frequency ratio $r$ is portrayed in Figure 5 . It is seen that SWD provides a SNR gain of approximately $4.8 \mathrm{~dB}$ that is practically independent of the sampling rate. For the TWD scheme, the SNR gain is marginally higher than that provided by SWD if $r>12$. As $r$ is decreased from 12 to 2 , the SNR gain of TWD falls sharply from $5.2 \mathrm{~dB}$ to $-1 \mathrm{~dB}$. It is clear that at lower sampling rates, the performance of the TWD scheme is very poor compared to that of the SWD scheme, while the SWD scheme performs consistently well at all sampling rates.

DOA estimation by MUSIC was done using undenoised, TWD denoised, and SWD denoised data. The variation of the bias and the mean square error (MSE) of the DOA estimate with SNR, is shown in Figures 6 and 7 . The bias and MSE were numerically computed by averaging over 100 Monte-Carlo simulations. The sampling frequency was chosen to be four times the source frequency. It is clearly seen that at lower SNRs, the bias and the MSE of the DOA estimates corresponding to the SWD denoised data are at least an order of magnitude less as compared to those corresponding to the TWD denoised data.

Figures 8 and 9 show the spectral MUSIC ambiguity function plots. The SNR of the received data was $-5 \mathrm{~dB}$. A total of $N=128$ temporal snapshots were used for performing SWD and calculating the spectral MUSIC ambiguity function. The three sources are clearly resolved for the spatially denoised data but they are not resolved when undenoised data is used, indicating a significant improvement in the resolution of the MUSIC estimator after SWD. 


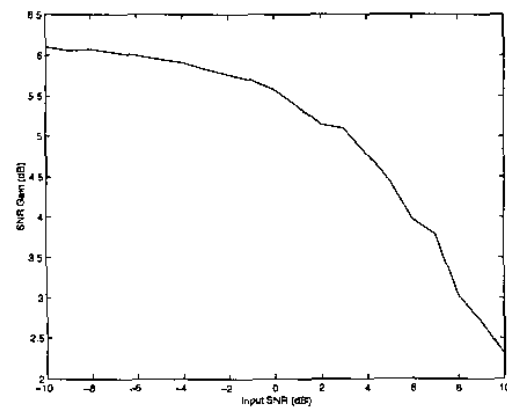

Figure 4: SNR Gain: SWD scheme.

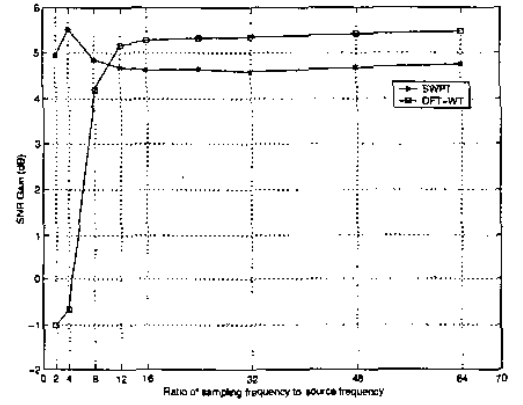

Figure 5: SNR Gain: SWD and TWD.

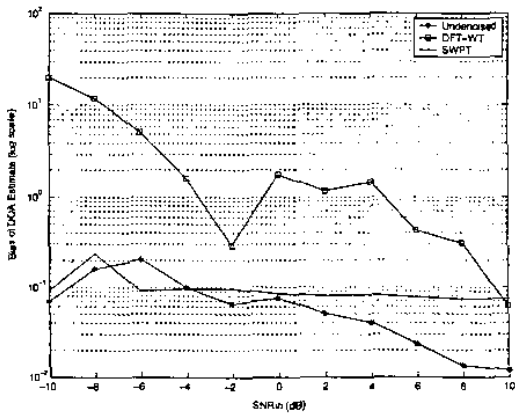

Figure 6: Bias of Estimate: $\theta=72^{\circ}$.

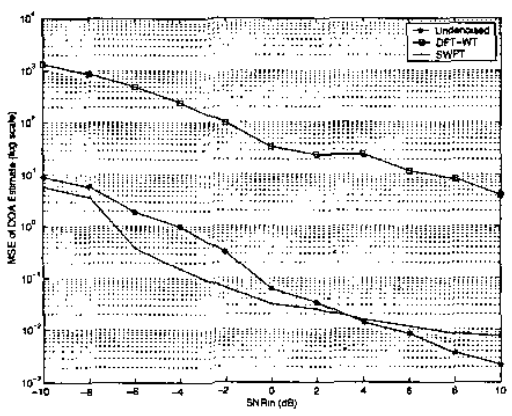

Figure 7: MSE of Estimate: $\theta=72^{\circ}$.

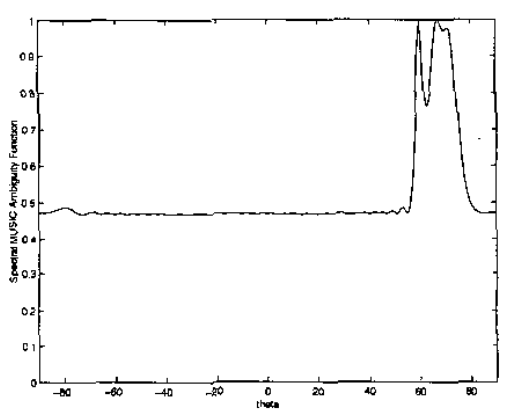

Figure 8: MUSIC: Undenoised Signal.

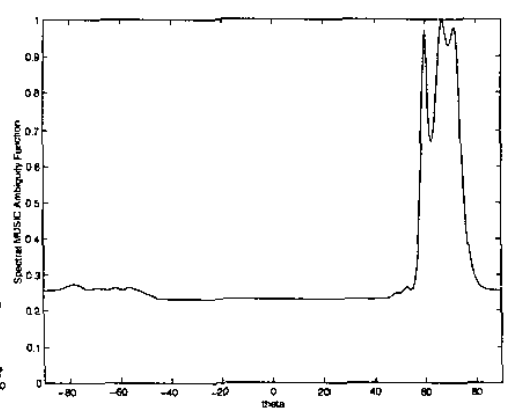

Figure 9: MUSIC: Denoised Signal. 


\section{CONCLUSIONS}

Plane wave signals received at a ULA can be denoised by performing a wavelet packet transform in the spatial domain and thresholding the resulting coefficients using an energy comparison algorithm. The method termed as Spatial Wavelet Denoising employs the SWPT and a block thresholding that selectively filters out noise in spatial frequency bands that do not contain signal. It is shown through simulations that SWD achives a SNR gain comparable to that of TWD at much lower sampling rates. SWD requires a sampling rate of just 2-4 times the signal frequency, whereas TWD requires a sampling rate of at least 12 times the signal frequency. Also, SWD does not involve the computation of spatial DFT and IDFT required by TWD based on the DFT-WT filter. Hence, SWD achieves a significant reduction in hardware complexity and computational complexity in comparison to TWD, without compromising the performance.

\section{ACKNOWLEDGEMENTS}

This work was partially supported by the DRDO-IISc program on Advanced Research in Mathematical Engineering.

\section{REFERENCES}

[1] D. L. Donoho, "De-noising by soft thresholding," IEEE Trans. Information Theory, vol. 41, pp. 613-627, 1995.

[2] D. L. Donoho and I. M. Johnstone, "Ideal spatial adaption via wavelet shrinkage," Biometrika, vol. 81, pp. 425-455, 1994.

[3] A. M. Rao and D. L. Jones, "A Denoising Approach to Multisensor Signal Estimation," IEEE Trans. Signal Processing, vol. 48, pp. 1225-1234, 2000.

[4] R. Sathish and G. V. Anand, "Wavelet Array Denoising for Improved Direction-of-Arrival Estimation," Technical Report No PME-2003-14, DRDO Program on Mathematical Engineering, Indian Institute of Science, Bangalore, October, 2003.

[5] R. Sathish and G. V. Anand, "Wavelet Denoising for Plane Wave DOA Estimation by MUSIC," presented at the IEEE-TENCON Conference, Bangalore, Karnataka, INDIA, October 14-17, 2003.

[6] R. O. Schmidt, "Multiple Emitter Location and Signal Parameter Estimation," in Proc. RADC of the 1979 Workshop on Spectral Estimation, Rome, NY, 1979, pp. 243-258.

[7] P. Stoica and R. Moses, Introduction to Spectral Analysis, NJ: Prentice-Hall Inc., 1997, ch. 4, pp. 139-180.

[8] C. S. Burrus, R. A. Gopinath, H. Guo, Introduction to Wavelets and Wavelet Transforms, NJ: Prentice-Hall Inc., 1998, Chp. 7, pp. 110-114.

[9] D. B. Percival and A. T. Walden, Wavelet Methods for Time Series Analysis, Cambridge University Press, 2000, Chp. 6, pp. 206-226. 